\title{
NONUNIFORM TEMPORAL ALIGNMENT OF SLICE SEQUENCES FOR FOUR- DIMENSIONAL IMAGING OF CYCLICALLY DEFORMING EMBRYONIC STRUCTURES
}

\author{
Michael Liebling, Julien Vermot, Arian S. Forouhar, \\ Morteza Gharib, Mary E. Dickinson, Scott E. Fraser ${ }^{1}$
${ }^{1}$ Biological Imaging Center, Beckman Institute, California Institute of Technology, Pasadena, CA
${ }^{2}$ Option of Bioengineering, California Institute of Technology, Pasadena, CA
${ }^{3}$ Dept. of Molecular Physiology and Biophysics, Baylor College of Medicine, Houston, TX

\begin{abstract}
The temporal alignment of nongated slice-sequences acquired at different axial positions in the living embryonic zebrafish heart permits the reconstruction of dynamic, three-dimensional data. This approach overcomes the current acquisition-speed limitation of confocal microscopes for real-time three-dimensional imaging of fast processes. Current synchronization methods align and uniformly scale the data in time, but do not compensate for slight variations in the heart rhythm that occur within a heartbeat. Therefore, they impose constraints on the admissible data quality. Here, we derive a nonuniform registration procedure based on the minimization of the absolute value of the intensity difference between adjacent slice-sequence pairs. The method compensates for temporal intra-sample variations and allows the processing of a wider range of data to build functional, dynamic models of the beating embryonic heart. We show reconstructions from data acquired in living, fluorescent zebrafish embryos.
\end{abstract}

\section{INTRODUCTION}

With speeds that reach several millimeters per second, heartwall motions and blood flow in the embryonic heart require fast frame-rates to be imaged under the microscope without inducing motion artifacts such as blurring or aliasing [1]. Confocal microscopes enable selective, three-dimensional imaging of fluorescent cells at depths up to $100-200 \mu \mathrm{m}$. Retrieving the temporal evolution of fluorescently labeled cells or blood flow gives invaluable insights for understanding the driving forces behind the development of the cardio-vascular system in early embryos.

Although recently developed confocal microscopes can capture images of two-dimensional slices at frame-rates as fast as 120 frames per s for $512 \times 512$ pixel images, direct recording of dynamic, three-dimensional data at similar framerates is currently not possible. To overcome this limitation,

Corresponding author is M. Liebling, California Institute of Technology, Mail Code 139-74, Pasadena, CA 91125, USA, email: liebling@ caltech.edu. (b)

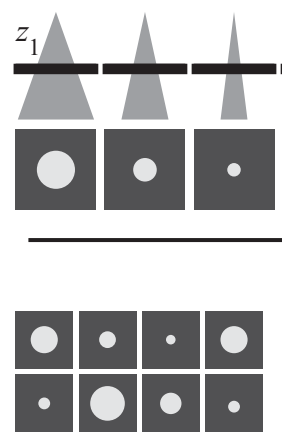

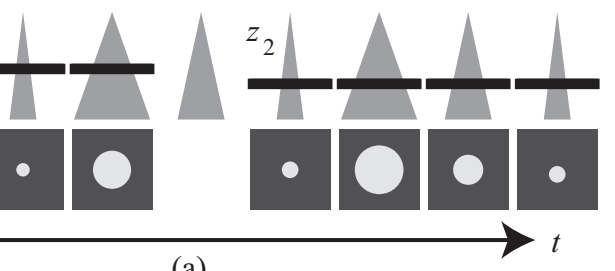

(a)
Fig. 1. Four-dimensional imaging: acquisition and reconstruction procedure. (a) Sequential acquisition of twodimensional slices (confocal microscopy) as a time-series at increasing depths from a cyclically deformed object (cone). (b) Direct reconstruction is not possible from the nongated data. (c) Temporal alignment procedure (d) Reconstruction.

an acquisition and reconstruction procedure based on the sequential, nongated acquisition of slice-sequences during several heart-beats and subsequent synchronization (see Fig. 1) was recently proposed [2]. In that work, the registration resulted from scaled, temporal shifting of the slice sequences. This technique is efficient if, during a single two-dimensional temporal sequence acquisition, the motion is periodic. The reconstruction precision and quality eventually depend on the conformity of the measurements to this hypothesis. From our observations, we noticed that slight irregularities (variation in the heart beat) of $1 \%$ can induce severe visual artifacts that prevent us from building accurate models and also force us to discard measurements that do not comply with the regularity hypothesis.

Here, we propose a nonuniform time-registration method that recursively aligns pairs of slice-sequences to build a fourdimensional reconstruction of the beating heart. The method is based on the minimization of the absolute intensity difference of the warped sequences' two-dimensional spatial wavelet coefficients, an optimization problem that we solve in a dynamic programming framework. 
While, to the best of our knowledge, the use of dynamic programming to solve our image reconstruction problem is new, similar registration techniques are widespread for different purposes, in particular, for speech analysis [3, 4].

In Section 2 we describe the proposed acquisition and reconstruction method. In Section 3 we present reconstructions that we obtained from in vivo imaging of fluorescent zebrafish embryos. In Section 4 we discuss our results and future research directions.

\section{METHOD}

\subsection{Acquisition Model and Cost Function Definition}

We model the measured intensity $I_{m}$ as follows,

$I_{m}\left(\mathbf{x}, z_{k}, t\right)=\iiint I\left[\mathbf{x}^{\prime}, z, \tau_{k}(t)\right] h\left(\mathbf{x}-\mathbf{x}^{\prime}, z_{k}-z\right) \mathrm{d} \mathbf{x}^{\prime} \mathrm{d} z$,

where $\tau_{k}(t)$ is an unknown warping function at depth $z_{k}=$ $k h_{z}, k=0, \ldots, N_{z}$. The warping functions $\tau_{k}: \mathbf{R}_{+} \rightarrow$ $\mathbf{R}_{+}, t \mapsto \tau_{k}(t)$ are strictly increasing, i.e. if $t_{1}<t_{2}$, we have $\tau_{k}\left(t_{1}\right)<\tau_{k}\left(t_{2}\right)$. The optical system's point spread function is denoted $h(\mathbf{x}, z)$. We assume that the four-dimensional intensity function $I(\mathbf{x}, z, \tau)$, has a period $T$, i.e.,

$$
|I[\mathbf{x}, z, \tau]-I[\mathbf{x}, z, \tau+T]| \ll I_{\max }
$$

We assume that at depth $z_{\bar{k}}$, the corresponding warping function is identity, i.e. $\tau_{\bar{k}}(t)=t$.

In order to recover an estimate of the volume $I(\mathbf{x}, z, \tau)$, $\tau \in[0, L], L>T$ from the measurements $I_{m}\left(\mathbf{x}, z_{k}, t\right)$, where $t \in\left[t_{k}, t_{k}+L^{\prime}\right]$ with $L^{\prime}>\sigma L(\sigma>1$ is the maximal stretching factor), we consider the following objective criterion to measure the discrepancy between the warped data from neighboring depths $z_{k}$ and $z_{k^{\prime}}$,

$$
\begin{aligned}
Q_{k, k^{\prime}, w_{k}}\left\{w_{k}^{\prime}\right\}=\int_{0}^{L} & \iint_{\mathbf{R}^{2}} \mid I_{m}\left[\mathbf{x}, z_{k}, w_{k}(\tau)\right] \\
& -I_{m}\left[\mathbf{x}, z_{k^{\prime}}, w_{k^{\prime}}(\tau)\right] \mid \mathrm{d} \mathbf{x} \mathrm{d} \tau .
\end{aligned}
$$

We aim at recovering the warping functions $w_{k^{\prime}}(\tau)$ for $k^{\prime}=$ $\left\{0, \ldots, N_{z}-1\right\} \backslash\{\bar{k}\}$. We start from the depth $z_{\bar{k}}$ for which $w_{\bar{k}}$ is known (identity) and find the functions $w_{k^{\prime}}$ recursively by aligning neighboring pairs, i.e. $\left(k, k^{\prime}=k-1\right)$ for pairs that are below $\bar{k}$ and $\left(k, k^{\prime}=k+1\right)$ for pairs above the reference depth $\bar{k}$. The reconstructed, dynamic volume is computed by warping the measurements, viz.

$$
I_{R}\left(\mathbf{x}, z_{k}, \tau\right)=I_{m}\left(\mathbf{x}, z_{k}, w_{k}(\tau)\right)
$$

To find the appropriate functions $w_{k}$ in practice, we proceed as follows. We consider discrete measurements (in space and time): for the image sequence that is to be aligned, $z_{k^{\prime}}$, we consider measurements taken at times $t=i h_{t}$, with $i=$ $0, \ldots, N_{t}-1$ and, for the reference sequence at depth $z_{k}$,
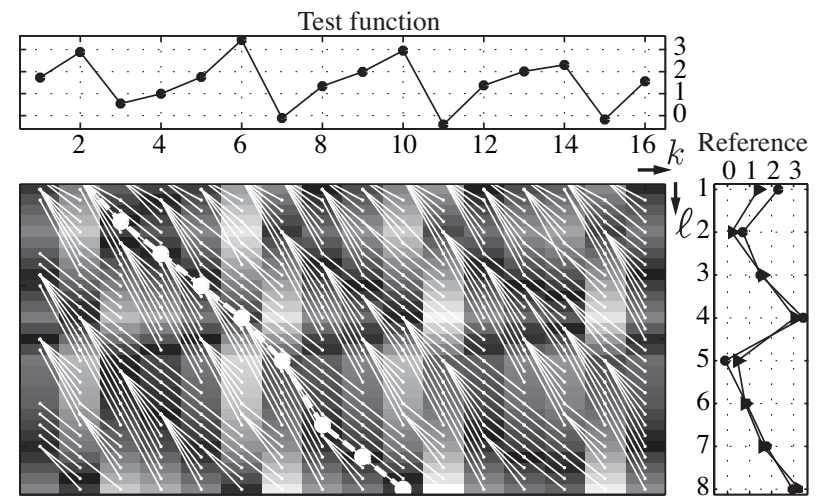

Fig. 2. Nonuniform temporal alignment via dynamic programming. A matrix is generated by taking the absolutedifference between samples of a test function (top) and a reference function (right, triangles). Proceeding recursively from left to right, a cost is associated to each position in the matrix, based on the value of the matrix at that position, the cost computed at the most favorable position in the neighboring left column (yielding a vertex), and a possible penalty if the pick induces stretching or compression of the time axis. Next, starting from locations on the bottom line, the path with lowest cost that reaches the top is kept, yielding the optimal warping function (bold) to interpolate the test function (right, circles).

measured at times $\tau=i h_{\tau}$, with $i=0, \ldots, \bar{N}_{r}-1$, we consider interpolated samples at times $\tau=i h_{\tau} / s$, with $i=$ $0, \ldots, N_{r}, N_{r}=\left(s \bar{N}_{r}\right)-1$ and $s \in \mathbb{N}_{+}^{*}$ an over-sampling factor (typically, $s=4$ ). The (discrete) problem boils down to finding the appropriate path in a two-dimensional graph, that pairs the test samples with the interpolated reference samples.

\subsection{Dynamic Programming for Temporal Alignment}

We denote $\mathbf{I}_{r}[\ell]$ the discrete, interpolated reference slice sequence and $\left.\mathbf{I}_{t}[k]\right)$ the test slice sequence. We compute the difference matrix $\Delta[\ell, k]=\left\|\mathbf{I}_{r}[\ell]-\mathbf{I}_{t}[k]\right\|$, with $k=0, \ldots, N_{t}-$ 1 and for $\ell=0, \ldots, N_{r}-1$, and where the norm $\|\cdot\|$ corresponds to a discretized version of Eq. (3). Exploring each of the $\left(N_{r}\right)^{N_{t}}$ possible paths across the matrix and finding the one that minimizes Eq. (3) would be of prohibitive complexity and possibly yield solutions that do not comply with the monotonicity hypothesis. In order to limit the number of paths to be considered, we use a dynamic programming [5] approach, resulting in a complexity $\mathcal{O}\left(N_{r} N_{t}\right)$.

We define $K\left(\ell, \ell^{\prime}\right)=\alpha\left(\left|\ell^{\prime}-\ell-w_{0}\right|\right)^{\gamma}+K_{\min }, \alpha, \gamma \in \mathbf{R}_{+}^{*}$. The limits for stretchability are given by $w_{\min } \leq w_{\max }<0$, $w_{\min }, w_{\max } \in \mathbb{Z}^{-}$. We recursively compute, for $k=0, \ldots, N_{t}$ 1 and for $\ell=0, \ldots, N_{r}-1$, the following quantities:

$$
L[\ell, k]= \begin{cases}L[\ell, k-1]+1 & \text { if } \ell>0 \text { and } k>0 \\ 1 & \text { otherwise }\end{cases}
$$




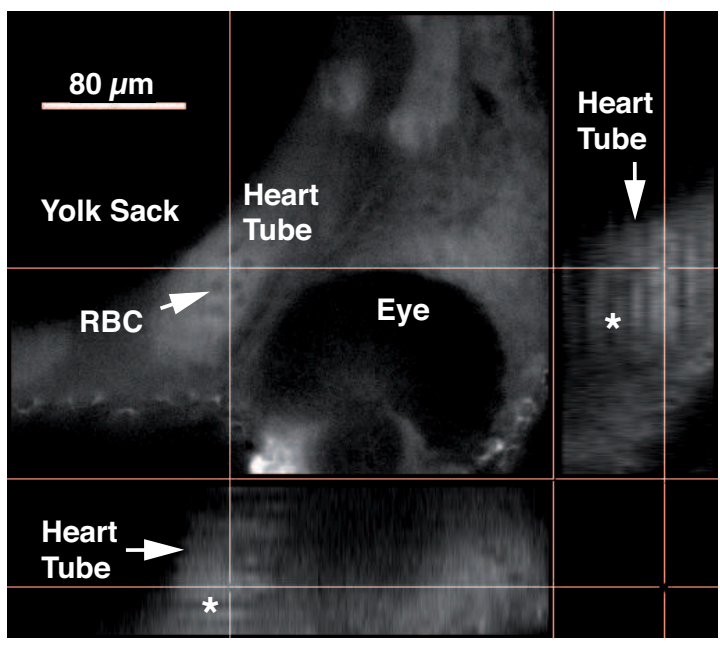

(a)

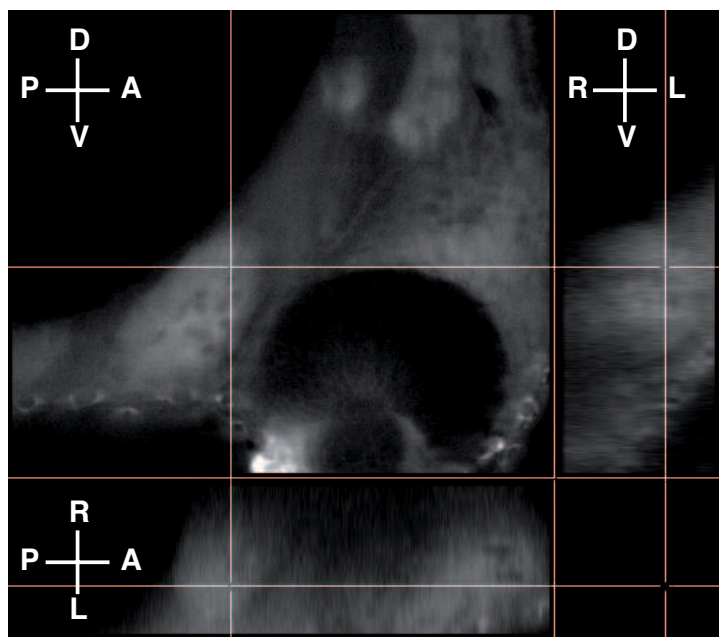

(b)

Fig. 3. Orthogonal slice views of a 38 h.p.f. zebrafish heart reconstruction. (a) Before alignment, temporally misaligned slices result in black streaks in the heart tube sections (two such slices are indicated by asterisks $*$ ). (b) After alignment, the heart tube can be recognized. Red blood cells (RBC) appear as black dots inside the heart tube. Movies are available online [6].

$$
\begin{gathered}
g[\ell, k]=\min _{\ell^{\prime}=\ell+w_{\min }, \ldots, \ell+w_{\max }} g^{\prime}\left(k, \ell, \ell^{\prime}\right), \\
\bar{\ell}[\ell, k]=\arg \min _{\ell^{\prime}=\ell+w_{\min }, \ldots, \ell+w_{\max }} g^{\prime}\left(k, \ell, \ell^{\prime}\right),
\end{gathered}
$$

that is, the length of the path, a cost function, and the index of the most favorable left neighbor, respectively, and where

$$
\begin{aligned}
& g^{\prime}\left(k, \ell, \ell^{\prime}\right) \\
& =\left\{\begin{array}{cc}
\left(g\left[\ell^{\prime}, k-1\right] L\left[\ell^{\prime}, k-1\right]\right. & \\
\left.+(1-\lambda) \Delta[\ell, k]+\lambda K\left(\ell, \ell^{\prime}\right)\right) & \text { if } \ell^{\prime} \geq 0 \text { and } k>0 \\
/\left(L\left[\ell^{\prime}, k-1\right]+1\right) & \text { if } \ell^{\prime} \geq 0 \text { and } k=0 \\
(1-\lambda) \Delta[\ell, k]+\lambda K_{\min } & \text { if } \ell^{\prime}<0 \\
(1-\lambda) \Delta[\ell, k]+\lambda K_{\min } & \text { and } \ell^{\prime}-\ell=w_{0} \\
\infty & \text { otherwise. }
\end{array}\right.
\end{aligned}
$$

The weighting factor $\lambda$ permits to adjust the warping stiffness. From the resulting graph (see Fig.2), we determine the lead of the optimal path by finding the vertex on the lower image border with the lowest cost and that yields an acceptable path:

$$
k_{0}=\arg \min _{k^{\prime} \in \mathcal{B}} g\left[N_{r}-1, k^{\prime}\right]
$$

with $\mathcal{B}=\left\{k^{\prime} \mid 1<k^{\prime}<N_{t}\right.$, and $\left.\tilde{\ell}_{k^{\prime}}[0] \leq 0\right\}$ and where the paths $\left\{\tilde{\ell}_{k^{\prime}}[k]\right\}_{0 \leq k<N_{t}}$ are defined for $1<k^{\prime}<N_{t}$

$$
\tilde{\ell}_{k^{\prime}}[k]= \begin{cases}N_{r}-1 & \text { if } k=k^{\prime} \\ \bar{\ell}\left[\tilde{\ell}_{k^{\prime}}[k+1], k^{\prime}+1\right] & \text { if } 0 \leq k<k^{\prime} \\ & \text { and } \tilde{\ell}_{k^{\prime}}[k+1] \geq 0 \\ 2 \tilde{\ell}_{k^{\prime}}[k-1]-\tilde{\ell}_{k^{\prime}}[k-2] & \text { if } k^{\prime}<k<N_{t} \\ 2 \tilde{\ell}_{k^{\prime}}[k+1]-\tilde{\ell}_{k^{\prime}}[k+2] & \text { otherwise. }\end{cases}
$$

The optimal path is then given by $\left\{\left(k_{0}-i, \tilde{\ell}_{k_{0}}[i]\right)\right\}_{0<i<N_{t}}$ unless $\mathcal{B} \equiv \emptyset$. The warping function $\bar{k}(j), j=s \ell$, and $\ell=0, \ldots, \bar{N}_{r}$ is obtained by inverting $\bar{\ell}_{k_{0}}(j)$ by use of linear interpolation.

Similarly to the approach taken in [2], we consider a limited set of wavelet coefficients instead of pixel values to compute the norm (3). We therefore ensure that the method is robust and that the computation of the difference matrix is fast.

\section{RESULTS}

Wild-type zebrafish (danio rerio) eggs were spawned using standard techniques [7] and the 38 hours post fertilization (h.p.f) old embryos were soaked in a green fluorescent dye (BODIPY FL $\mathrm{C}_{5}$-ceramide, Molecular Probes) for 3 hours. We acquired two-dimensional image sequences at 60 frames per $\mathrm{s}$ for $2 \mathrm{~s}$, corresponding to 3-4 heartbeats, using a fast slit-scanning confocal microscope (Zeiss LSM 5 LIVE, Carl Zeiss Jena GmbH, Germany) with 488nm excitation light and a 500-525nm band-pass filter to filter emission signal. Between the acquisition of two sequences, the stage was moved axially by $10 \mu \mathrm{m}$ or $5 \mu \mathrm{m}$, depending on the magnification of the microscope objective $(10 \times$ or $40 \times$, respectively). Per embryo, we could image a total of 20-30 depths. The images of $512 \times 512$ pixels had a sampling step of $1.78 \mu \mathrm{m} \times$ $1.78 \mu \mathrm{m}$ per pixel (Zeiss Plan-Neofluar $10 \times / 0.3$ microscope objective), or $0.62 \mu \mathrm{m} \times 0.62 \mu \mathrm{m}$ per pixel (Zeiss C-Apochromat $40 \times / 1.2 \mathrm{~W}$ microscope objective). The images were realigned by use of a MATLAB implementation of the method described above. The four-dimensional data was analyzed and visualized using appropriate software (Imaris, Biplane 

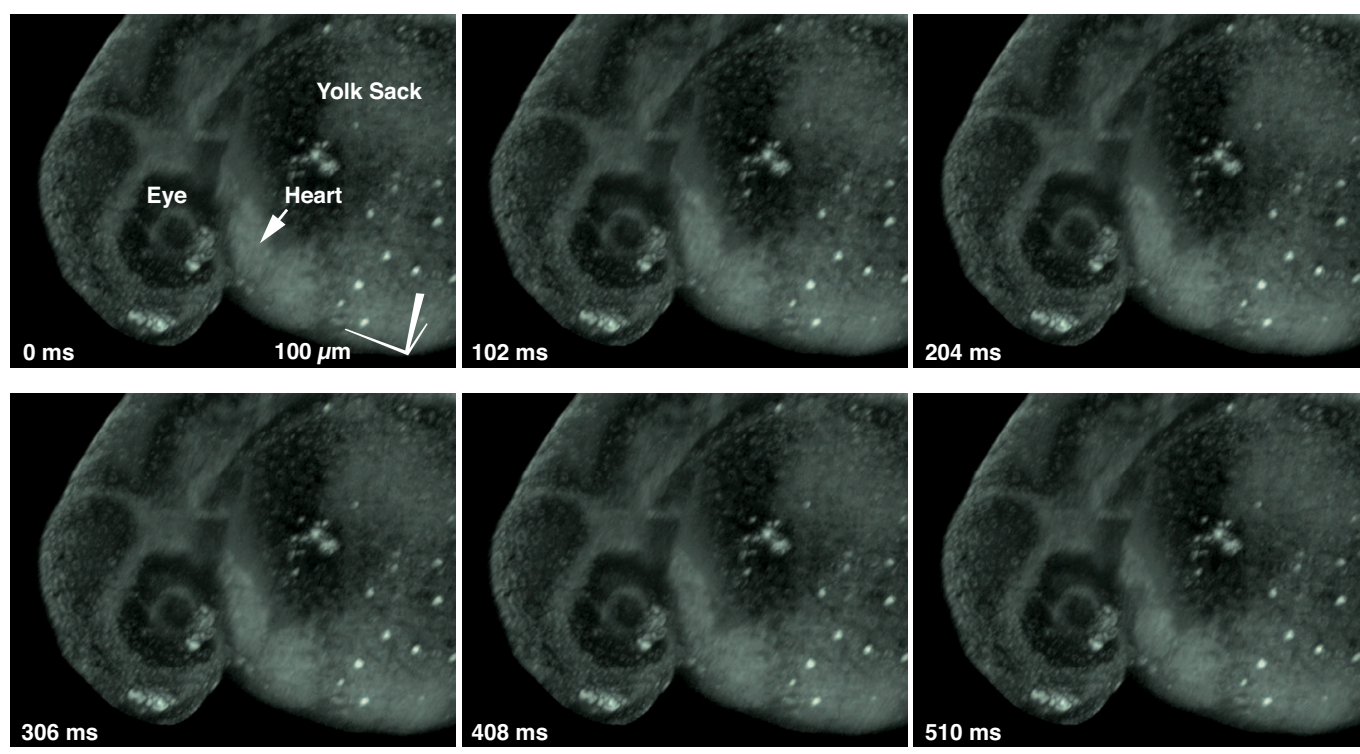

Fig. 4. Four-dimensional reconstruction of a 38 h.p.f living zebrafish. The heart-tube is visible in the center, pumping blood. The corresponding movie is available online [6].

AG, Zurich, Switzerland). The reconstructions prior and after alignment are presented in Fig. 3 (a) and (b), respectively. The reconstructed data can be rendered (see Fig. 4) and further analyzed.

\section{DISCUSSION AND CONCLUSION}

The presented non-uniform temporal alignment procedure allows reconstruction of four-dimensional volumes from slice sequences acquired in living zebrafish embryos, even in cases where the time series are not strictly periodical. This constitutes a major improvement over previously proposed techniques that only included uniform scaling and translation in time. We plan further investigations on the method, including extensive evaluation and comparisons on synthetic data as well as validation on a large body of experimentally acquired data. Possible extensions also include deconvolution, segmentation, and flow analysis of the reconstructed data.

\section{ACKNOWLEDGMENTS}

We thank Sean Megason, Le Trinh, and Chris Waters for help with zebrafish preparation and advice on imaging techniques. M.L. would like to thank Michael Unser for helpful discussions and for sharing unpublished work on related topics.

\section{REFERENCES}

[1] J. R Hove, R. W. Köster, A. S. Forouhar, G. AcevedoBolton, S. E. Fraser, and M. Gharib, "Intracardiac fluid forces are an essential epigenetic factor for embryonic cardiogenesis," Nature, vol. 421, pp. 172-177, Jan. 2003.

[2] M. Liebling, A. S. Forouhar, M. Gharib, S. E. Fraser, and M. E. Dickinson, "Four-dimensional cardiac imaging in living embryos via postacquisition synchronization of nongated slice sequences," J. Biomed. Opt., vol. 10, no. 5, pp. 054001 1-10, 2005.

[3] H. Sakoe and S. Chiba, "Dynamic-programming algorithm optimization for spoken word recognition," IEEE Trans. Acoust., Speech, Signal Processing, vol. 26, no. 1, pp. 43-49, 1978.

[4] C. Yang and M. Stone, "Dynamic programming method for temporal registration of three-dimensional tongue surface motion from multiple utterances," Speech Commun., vol. 38, no. 1, pp. 201-209, 2002.

[5] R. Bellman, Dynamic Programming, Princeton Univ. Press, Princeton NJ, USA, 1957.

[6] http://bioimaging.caltech.edu/publications/isbi2006/.

[7] M. Westerfield, The Zebrafish Book, University of Oregon Press, Eugene, 1995. 